\title{
El patrimonio geológico y paleontológico en la región de Aragón
}

\author{
Guillermo Meléndez, Alizia Nuñez, Marta Tomás | Dpto. de Paleontología, Universidad de Zaragoza \\ Olga López Guajardo, Javier Lorente Obón | Empresa QUETEO \\ Monserrat Soria | profesora de Secundaria del Gobierno de Aragón \\ Paola Infante | Asesora Técnica en la Dirección General de Ordenación del Territorio del Gobierno de Aragón
}

URL de la contribución <www.iaph.es/revistaph/index.php/revistaph/article/view/4183>

El marco legal sobre patrimonio geológico en Aragón

Durante los años 80, mientras el Estado Español se iba reconstruyendo en diferentes comunidades autónomas, la región aragonesa comenzó un trabajo de reformas y elaboración de un marco legal de reconocimiento y protección de su patrimonio cultural, geológico y paleontológico. Este proceso se prolongó durante bastantes años y fue perfilándose poco a poco durante las dos siguientes décadas. Dicha tarea no resultó sencilla. A la complejidad que ya de por sí tenía esta labor debido a la magnitud del proyecto, se le sumaba que no siempre existió un consenso total entre todas las instituciones implicadas, concretamente acerca del procedimiento ideal o más eficiente a seguir, lo cual alargó el proceso más tiempo del estimado en un principio. Posteriormente la situación mejoró, especialmente con la iniciativa del Instituto Geológico y Minero de España (IGME) de generar una legislación más acorde con la existente en cada región que facilitó la tarea de las distintas áreas y regiones de España.

No obstante, todavía quedaban por perfilar ciertas cuestiones. En la sede del Gobierno de Aragón se instaló una serie de oficinas lideradas por personas escasamente relacionadas con el patrimonio paleontológico. Cuando solicitaron ayuda a la Universidad, encontraron con investigadores que tenían visiones muy diferentes sobre el patrimonio geológico y paleontológico. El resultado fue problemático y confuso, ya que el patrimonio paleontológico no se incluyó dentro del patrimonio geológico. Por suerte para los paleontólogos, el IGME se encontraba elaborando una guía sobre los valores patrimoniales de las distintas regiones españolas y el patrimonio paleontológico ocupaba un lugar destacado junto con otros valores patrimoniales (por ejemplo, el patrimonio histórico y el estratigráfico).

Sin embargo, a pesar de que en los catálogos del IGME el patrimonio paleontológico ocupa un lugar notable, en la sede del Gobierno de Aragón su situación era escasamente clara. Pese a ello, parte de las autoridades no han tenido inconveniente en achacar a los investigadores las culpas de su deficiente gestión. El resultado ha sido la ruptura de relaciones entre la Administración y los investigadores.

En las páginas siguientes ofrecemos una visión detallada (en la medida de lo posible) de la situación actual del patrimonio paleontológico en la región aragonesa. Como podremos ver, éste es enormemente importante por todos los campos que abarca aunque no siempre se encuentre reconocido como debiera. No obstante, pensamos que ésta es una muy buena ocasión para mostrar su enorme riqueza y diversidad.

\section{El cabalgamiento de Daroca}

En la localidad de Daroca, a unos $80 \mathrm{~km}$ al sur de Zaragoza, los materiales del Cámbrico cabalgan sobre los del Mioceno Superior. Este cabalgamiento fue reconocido ya en los años 20 por Franz Lotze, quien en su tesis doctoral (1929) lo señalaba como una probable evidencia del empuje final de la placa africana durante la orogenia alpina.

En 1943, Bermudo Meléndez, en su tesis doctoral, estudió el yacimiento de trilobites de Murero, muy cerca de Daroca y también mencionó este cabalgamiento. Su interpretación fue similar a la de Lotze. Los dos auto- 
a debate El marco legal para la protección del patrimonio paleontológico. ¿Qué pasa en tu comunidad?

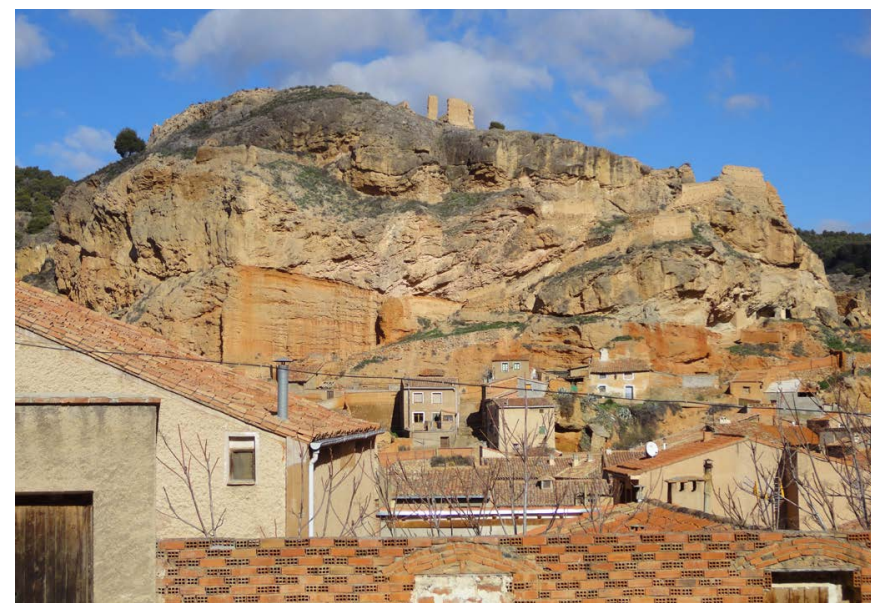

Cabalgamiento de Daroca. Obsérvese cómo los materiales del Cámbrico cabalgan sobre los materiales del Mioceno. Un poco más adelante se puede reconocer a los materiales del Mioceno cabalgando sobre sí mismos, lo cual es una clara evidencia de la intensidad de la Orogenia alpina | foto de Andrés Gil Imaz

res coincidieron en afirmar que se podría tratar de una "cobijadura" tectónica sin grandes desplazamientos. Posteriormente el catedrático de la Universidad de Asturias J. Albareda aportó nuevas evidencias y coincidió con estos autores en decir que era una cobijadura sin grandes desplazamientos, aunque era cierto que en las proximidades se encontraban abundantes muestras de que el desplazamiento podría haber sido más importante de lo supuesto en los inicios.

En pocos años este cabalgamiento pasó a tener un interés preferente dentro del marco legal de la protección del patrimonio geológico aragonés, por lo que se incluyó como punto de especial interés geológico (PIG) en la cordillera ibérica. Mostraba claramente lo que había sido el resultado del empuje de la placa africana sobre el bloque ibérico al final del Mioceno (en el Messiniense). En realidad, el Cámbrico monta sobre los materiales del Mioceno Superior produciendo lo que se denomina una "inversión tectónica". Este empuje no sólo provocó este cabalgamiento sino que generó el cabalgamiento de los materiales del Mioceno sobre sí mismos. En efecto: si se pasa del valle del Jiloca al valle del Río Peregiles, se puede ver perfectamente cómo los materiales del Mioceno montan sobre sí mismos y dejan un desnivel de más de 40 metros entre ellos. En la actualidad, este cabalgamiento ha pasado a ser un punto fundamental de interés geológico, paleontológico y didáctico-turístico de esta región y los geólogos lo incluyen como punto de interés preferente, por lo que goza de una especial protección. Se puede decir que ha pasado a constituir una de las más sólidas evidencias empíricas de la tectónica alpina en la cordillera ibérica, referente fundamental para reconstruir su historia geológica (y de todos los orógenos españoles). En los últimos años este cabalgamiento ha pasado a tener un tratamiento preferente entre los puntos más protegidos de la región aragonesa y goza de una protección especial, siendo un referente fundamental del marco legal de esta región. Su interés ha llevado a que sea incluido como punto de especial interés geológico en el recorrido turístico-didáctico que se hace anualmente, el denominado Geolodía.

\section{El Parque Nacional de Ordesa y Monte Perdido}

Con más de 15.000 ha de superficie, el Parque Nacional de Ordesa y Monte Perdido se posiciona como la figura de protección más importante del territorio aragonés. Localizado en la parte más septentrional de la provincia de Huesca en la frontera con Francia, fue declarado el 16 de agosto de 1976 mediante Real Decreto y posteriormente ampliado en 1982 hasta alcanzar su actual superficie. A pesar de tratarse de una figura de protección de ámbito nacional, su gestión se traspasó a la comunidad autónoma en 2006, y en 2015 el Gobierno de Aragón aprobó el último Plan Rector de Uso y Gestión (en adelante, PRUG) el cual es utilizado como actual instrumento básico de planificación.

Que ostente la figura de más alto rango de protección está ampliamente justificado pues en este espacio confluyen otras muchas figuras: monumento natural de los glaciares pirenaicos, espacios protegidos de la Red Natura 2000 (LIC y ZEPA), reserva de la biosfera, patrimonio de la humanidad de la Unesco o geoparque mundial de la Unesco, entre otros.

En lo que respecta al patrimonio geológico y paleontológico, el PRUG es claro al considerar incompatible 
a debate El marco legal para la protección del patrimonio paleontológico. ¿Qué pasa en tu comunidad?

cualquier alteración o sustracción de rocas, minerales y fósiles. No obstante, así como existe información sobre fauna y flora a través de la cartelería del parque, se observa la ausencia de referencias a la geología, por lo que difícilmente los visitantes pueden ser conscientes del valor del entorno.

En este sentido, cabe destacar que el patrimonio geológico presente en ese parque nacional acumula numerosos y llamativos ejemplos, como los valles glaciares de Ordesa y Pineta o los cañones fluviokársticos de Añisclo o Escuaín, valles colgados como el de Cotatuero o ventanas tectónicas como la de La Larri. El registro fósil es amplio y es fácil encontrar restos fósiles de espon-

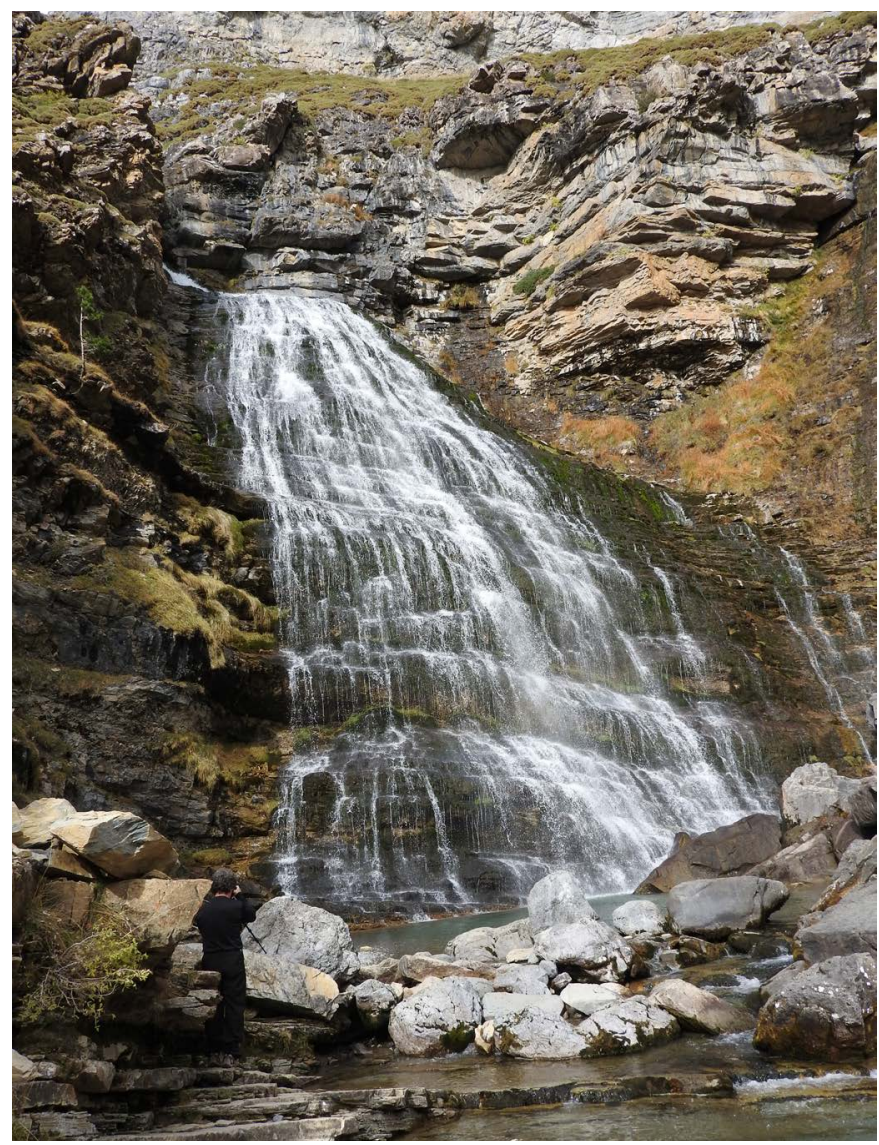

Cascada de la Cola de Caballo, Parque Nacional de Ordesa. Este es uno de los puntos de mayor interés del Valle de Ordesa | foto Paola Infante jas, foraminíferos, rudistas, equinodermos o corales. Es conocido el hallazgo de un cráneo de cocodrilo marino que está pendiente de estudio.

Todo ello hace de este lugar uno de los mejores para comprender la formación de los Pirineos y donde pueden observarse señales de las dos orogenias que ha sufrido esta zona: una primera fase de plegamiento que tuvo lugar durante el Pérmico (Orogenia Hercínica), que conformó una primitiva cordillera de la que todavía se pueden encontrar señales en los materiales más antiguos del Pirineo, y una segunda que comienza en el Triásico y se extiende hasta el Cretácico Superior y el Terciario, extendiéndose por gran parte del Cenozoico (Orogenia Alpina) y que es la responsable en gran parte de los relieves actuales, los cuales han sido profundamente modelados durante los episodios de glaciaciones más recientes.

En definitiva, el valor del Parque Nacional de Ordesa y Monte Perdido desde el punto de vista patrimonial es incalculable. Pese a la confluencia de numerosas figuras de protección, la ausencia de información relativa a la geología de esta zona no impide que estos bienes pudieran resultar dañados, algo que podría evitarse favoreciendo la divulgación del valor de este patrimonio mediante la ampliación o modificación de la cartelería, la creación de rutas geológicas específicas o bien organizando actividades, jornadas...

\section{Patrimonio geológico para todos públicos del Parque Cultural del Río Martín}

Muchos lugares de interés geológico en Aragón catalogados como puntos o áreas de interés geológico y protegidos por el marco legal vigente en Aragón, la Ley de Patrimonio Natural y biodiversidad, la Ley del Patrimonio Cultural de Aragón y el Decreto de catalogación y protección del patrimonio geológico, se pueden considerar como auténticos recursos turísticos por su atractivo y singularidad.

El aprovechamiento de éstos en el sector turístico para difundir y divulgar la geología al público general es 


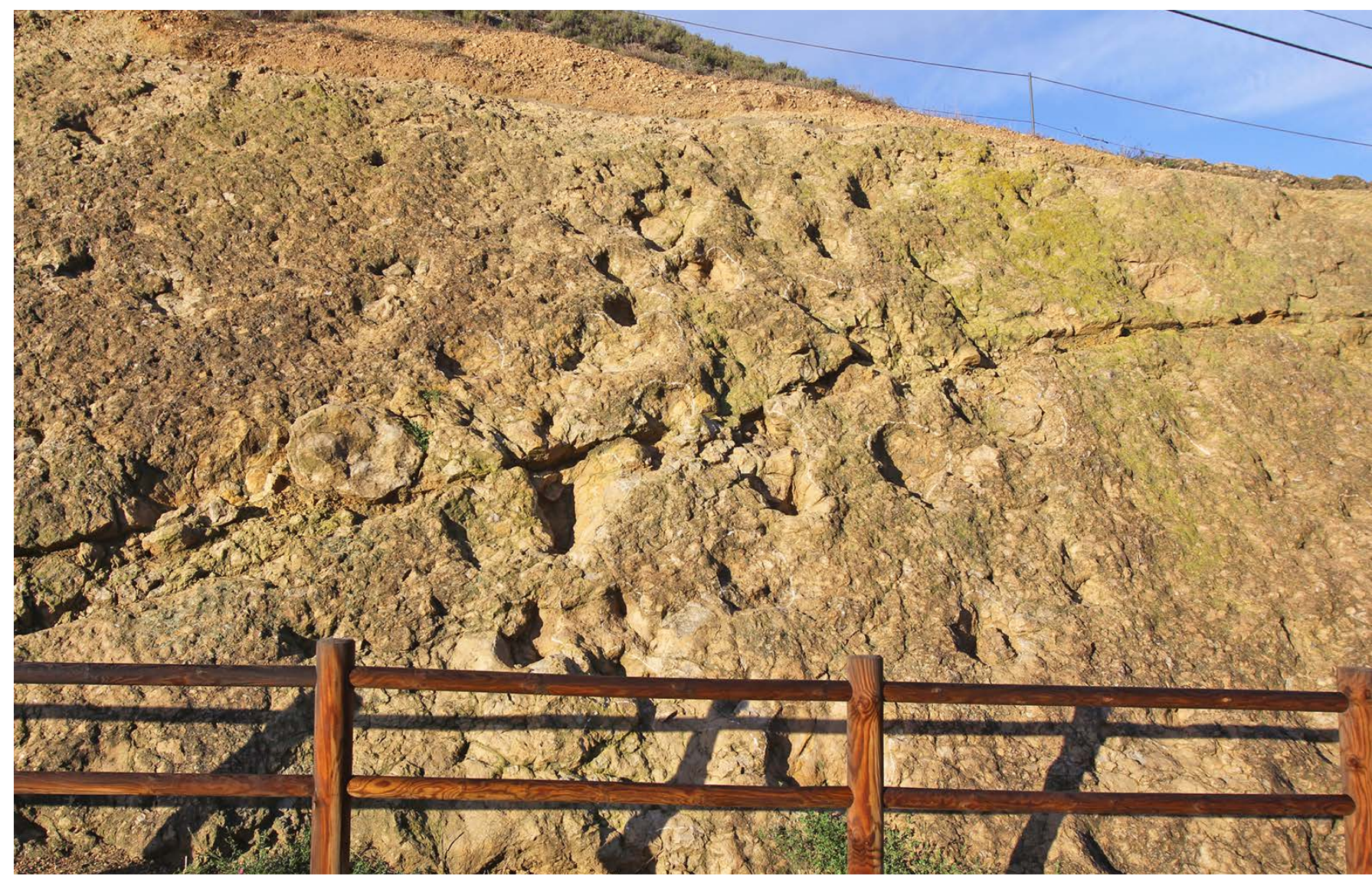

Yacimiento de icnitas del Río Escuriza, Ariño, Teruel. El encajamiento de las conchas de los ostreidos constituyen una evidencia irrefutable de que este depósito corresponde con la zona supramareal de la cuenca. Es decir estos ejemplares no se encuentran en el lugar donde vivían | foto Olga López y Javier Lorente

una iniciativa a tener en cuenta. El Plan Aragonés de Estrategia Turística 2016-2020 incluye al geoturismo y al turismo geológico como motores de productos turísticos innovadores. Este hecho favorece la creación de productos turísticos basados en el aprovechamiento sostenible del patrimonio geológico aragonés.

Cabe destacar que el marco legal insta también a difundir y divulgar el patrimonio geológico además de conservarlo y protegerlo, por lo que, desde las administraciones públicas, se está haciendo un esfuerzo para financiar actuaciones con tal fin.

La comunidad autónoma de Aragón cuenta con destinos ejemplares para potenciar el geoturismo, como es el caso del Parque Cultural del Río Martín, un área que aglutina un sinfín de recursos de diferente índole, incluyendo, según las leyes anteriormente mencionadas y la Ley de Parques Culturales de Aragón, los elementos geológicos en él existentes.

Algunos de los elementos geológicos que posee este parque cultural están catalogados según el decreto anteriormente mencionado como puntos de interés geológico (PIG). A continuación, se enumeran y se describen brevemente aquellos que cumplen con las premisas para ser recursos turísticos (singularidad, atractivo, estado de conservación, infraestructuras de apoyo interpretativo, accesibilidad, estacionalidad del recurso) y que, por lo tanto, son óptimos para ser visita- 
a debate El marco legal para la protección del patrimonio paleontológico. ¿Qué pasa en tu comunidad?

dos por los turistas o visitantes que llegan a esta zona sin conocimiento alguno sobre conceptos y procesos geológicos.

> Baños de Ariño. Idóneo para dar a comprender la circulación del agua subterránea y su salida al exterior de las rocas en forma de manantiales, fuentes y surgencias, que reflejan claramente la existencia de un plano de cabalgamiento que llegaría hasta profundidades notables, similar al de la zona de Alhama de Aragón.

> Sima de Oliete. Óptimo para dar a conocer los procesos de disolución de las rocas calizas y las morfologías resultantes.

$>$ Yacimiento de huellas de dinosaurios de Ariño. Apto para divulgar los procesos de fosilización y la reconstrucción del paleopaisaje del Cretácico Inferior. Algunos caracteres, tales como la desarticulación y el encajamiento de las conchas de los bivalvos son una clara evidencia empírica de que forman una serie de elementos encajados postmortalmente y que se encuentran en el supramareal. Las pisadas de los dinosaurios deformando estas conchas confirman esta afirmación.

$>$ Areniscas de Peñasroyas. Ejemplo para interpretar los procesos sedimentarios y petrogenéticos así como el paleoclima del Triásico Inferior.

> Pliegue de Alcaine. Muestra la generación de morfologías (pliegues y fallas) en el terreno debido a la energía interna, y los procesos de plegamiento, de la Tierra.

El diseño de un producto geoturístico se basa en la agrupación de estos recursos turísticos en forma de ruta de uno, dos o cuatro días, conformando así un paquete turístico. Como va dirigido a todo tipo de público se deben contemplar también las visitas a otros lugares relacionados con la historia, religión, salud, etnografía y gastronomía, de esta manera se asegura el éxito del producto enriqueciéndolo y dotándole de un mayor atractivo. El apoyo de profesionales del sector turístico (técnicos de turismo, agentes de viajes, consultores de marketing turístico, etc.) es fundamental para la comercialización este producto geoturístico y cualquier otro.

Concluimos entonces que:

$>$ La integración del patrimonio geológico en los planes estratégicos turísticos hace patente su transformación en recurso turístico, siendo, este hecho, una oportunidad para acercar al público general la Geología.

$>$ El patrimonio geológico aragonés se caracteriza por ser muy diverso lo que facilita generar productos turísticos sugerentes que muestren una variedad de procesos geológicos relativos a las diferentes ramas que conforman la Geología.

$>$ El marco legal aragonés hace alusión a la necesidad de su difusión y su divulgación por eso es necesario que desde las administraciones públicas se creen partidas presupuestarias para dichos objetivos.

$>$ El trabajo en red con los agentes turísticos del ámbito público y privado es fundamental para promocionar y comercializar el producto.

\section{Límite Calloviense-Oxfordiense en el sector Sierra de Arcos (Teruel)}

Uno de estos casos singulares se hace patente en los materiales del Grupo Chelva que representan el tránsito entre el Jurásico Medio y Superior en los entornos de las localidades aragonesas de Ariño, Andorra, Calanda y Alcorisa, en donde forman secuencias condensadas muy fosilíferas.

Los fósiles de ammonoideos, espongiarios, bivalvos, braquiópodos y demás invertebrados marinos, muestran un conjunto de caracteres tafonómicos que permiten reconstruir, por un lado, los procesos alterativos experimentados por las entidades conservadas $\mathrm{y}$, por otro, la dinámica evolutiva de la cuenca (tectónica de basculamiento de bloques) durante el Jurásico Medio y Superior, como evidencia el análisis de las poblaciones tafónicas registradas. 
a debate El marco legal para la protección del patrimonio paleontológico. ¿Qué pasa en tu comunidad?

Los ammonoideos presentes en estos niveles se corresponden con macroconchas adultas. Esta curiosa selección que presentan es un claro indicador de la aloctonía de los mismos. Tras el momento de producción biogénica, las conchas de mayor diámetro habrían sido transportadas por un proceso de deriva necroplanctónica hacia zonas más someras de la cuenca, debido a su mayor potencial de flotación, recorriendo mayor distancia que otros ejemplares de tamaños inferiores. Además los fósiles presentan un alto grado de encajamiento y los ammonites corresponden en la mayoría de los casos con ammonites huecos, indicando un proceso de enterramiento rápido y cementación temprana. Este depó-

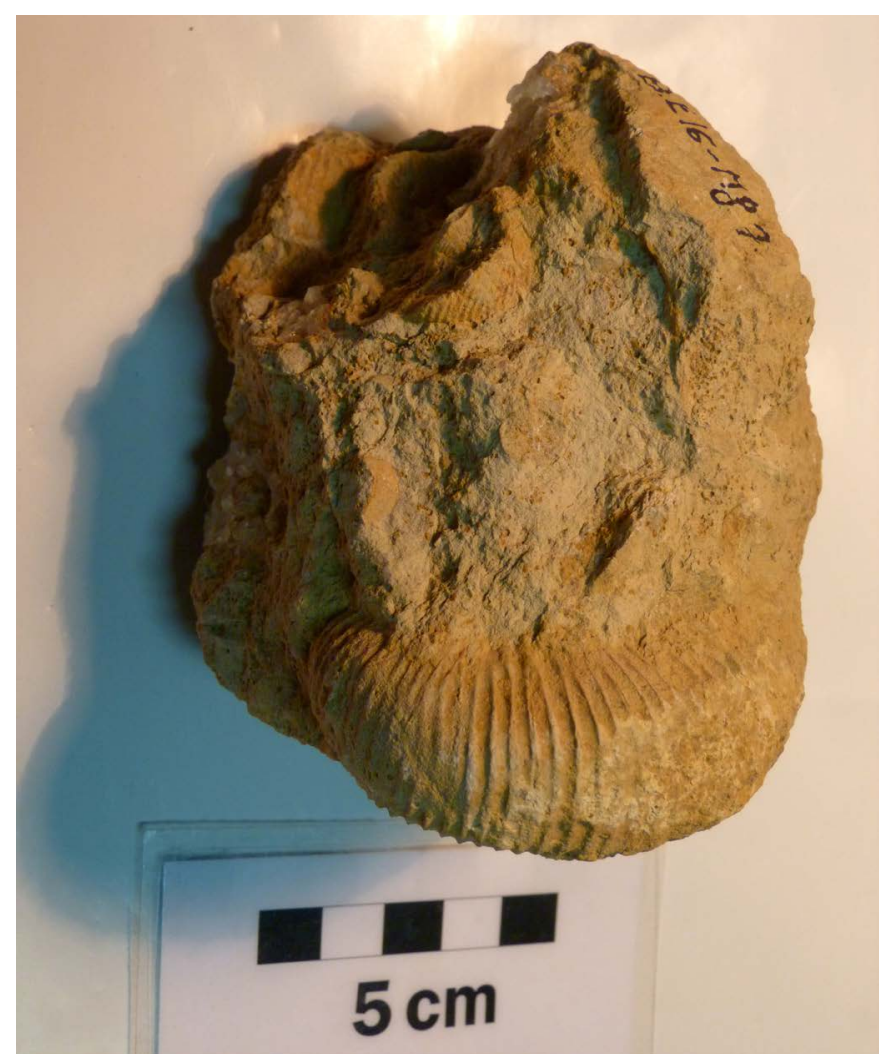

Ejemplar de Macrocephalites reelaborado del límite Calloviense-Oxfordiense de Ariño. Los criterios de reelaboración son claramente el relleno micrítico del ejemplar de ammonites (Macrocephalites), el grado de verticalización de las conchas y el hecho de estar envuelto en caliza oolítica, esta discontinuidad es una clara evidencia de la diacronía del ejemplar con el nivel que lo contiene | foto Guillermo Meléndez sito ha sido generado por acción de tormentas en un ambiente de plataforma carbonatada somera.

Por encima de esta unidad, en el tránsito entre los pisos Calloviense y Oxfordiense, se encuentra el nivel de oolitos ferruginosos de la capa de Arroyofrío, que refleja una laguna estratigráfica de amplitud variable, mayor en la parte distal y menor en la parte proximal de la plataforma. La discontinuidad se desarrolló debido a un proceso de exposición subaérea de este sector de la plataforma en ese momento, este hecho queda reflejado en la abundante presencia de ejemplares reelaborados que permiten reconocer un clino tafonómico por abrasión, con desarrollo de surcos anulares y desarticulación a favor de los septos. De acuerdo con algunos autores (MELÉNDEZ; BELLO; DELVENE et ál., 1997) estos niveles se encontraban durante el Jurásico Medio y Superior, formando parte de un alto paleogeográfico: el denominado "Alto de Ariño-Andorra" (AURELL, 1990; FONTANA; MELÉNDEZ, 1990).

La primera unidad del Jurásico Superior es la formación Yátova, que está representada, en su parte inferior, por calizas con espongiarios que muestran una clara cementación temprana, inversión y un alto grado de encajamiento (lo cual refleja probables episodios de tormenta a una cierta profundidad). Por el contrario, a partir de un cierto nivel estos materiales pasan a ser calizas glauconíticas. Éstas representan facies muy someras de la cuenca con una marcada influencia del oleaje. Otros caracteres, como el hecho de que se encuentren fósiles de Pholadomyidos semienterrados (cuando los Pholadomyidos son formas infaunales profundas), indican claramente un proceso de somerización y erosión entre los distintos episodios sedimentarios.

Tal como se ha indicado, hacia el este, las unidades del Jurásico disminuyen de espesor. En Andorra todo el Jurásico Medio supera apenas los veinte metros. El Oxfordiense no sobrepasa los cinco o seis metros y en Alcorisa su espesor es aún menor. Todo esto constituye una clara evidencia empírica de la acción de fallas lístri- 
a debate El marco legal para la protección del patrimonio paleontológico. ¿Qué pasa en tu comunidad?

cas que habrían generado el basculamiento de la plataforma durante esta parte del Jurásico, reflejando lo que sería la apertura del Tethys a lo largo de lo que comenzaría con el surco Liguro-Piamontes. Un claro indicador de este proceso lo constituye el hecho de que a continuación, por encima del Oxfordiense, los materiales del Kimmeridgiense superan ampliamente los 40 y 50 metros.

A la vista de los hechos expuestos, está claro que los procesos de basculamiento de la plataforma epicontinental quedan claramente reflejados en aspectos tales como la deriva de las conchas de ammonoideos, los caracteres tafonómicos de éstos, el grado de encajamiento de los espongiarios, las distintas facies y los cambios de espesor que se reconocen en los diferentes puntos descritos $y$, especialmente, el hecho de que las diferencias de espesor entre los materiales del Oxfordiense y Kimmeridgiense en Ariño y Calanda y la zona más externa (Alcorisa) son claros indicadores de la dinámica de bloques que predomina en la plataforma ibérica durante el Jurásico.

Tras bastantes años de polémicas y problemas con la Administración esta región ha pasado a ocupar un lugar preferente como lugar protegido, aunque sigue estando expuesto a la acción incontrolada de coleccionistas sin escrúpulos. Aún así mantiene el estatus de zona especialmente protegida como evidencia de los procesos sedimentarios ocurridos durante este período. Es un punto de interés geológico, didáctico y (sobre todo) turístico de gran relevancia. En la actualidad, muchos de los recorridos geológico-didácticos que se organizan suelen pasar por esta zona.

\section{BIBLIOGRAFÍA}

- AURELL, M. (1990) El Jurásico Superior de la Cordillera Ibérica central (provincias de Zaragoza y Teruel). Análisis de cuenca. Tesis doctoral, Universidad de Zaragoza, 1990

- FONTANA, B.; MELÉNDEZ, G. (1990) Caracterización bioestratigráfica de la Biozona Transversarium (Oxfordiense medio) en el sector oriental de la Cordillera Ibérica. Geogaceta, 8, 1990, pp. 76-78

- LOTZE, F. (1929) "Stratigraphie und Tektonik des keltiberischen Grundgebirges (Spanien)". Abhandlung des Gesichten und des Wissenschaftlichen. Göttingen MathPhys. KI. n.F. 14. (Beitrage das Geologischen und Westlichen Mediterraneansgebiete, Nr3). Berlin 1929

- MELÉNDEZ, B. (1943) El Cámbrico en la Península Ibérica. Tesis doctoral, Universidad Complutense de Madrid, 1943

- MELÉNDEZ, G.; BELLO, J.; DELVENE, G.; PÉREZURRESTI, I. (1997) EI Jurásico Medio y Superior (CallovienseKimmeridgiense) en el sector de la Llanura de Arcos (AriñoOliete, Teruel): Análisis tafonómico y Bioestratigrafía. Cuadernos de Geología Ibérica, Tafonomía y fosilización, 23, 1997, pp. 269-300

\section{Agradecimientos}

Nuestro sincero agradecimiento a Andrés Gil Imaz por habernos facilitado una foto del cabalgamiento de Daroca, que muestra claramente el avance de los materiales del Cámbrico sobre los del Mioceno. 